\title{
Models of Language Evolution
}

Cathleen $0^{\prime}$ Grady $^{1, *}$ and Kenny Smith ${ }^{1}$

${ }^{1}$ Centre for Language Evolution, School of Philosophy, Psychology and Language Sciences, University of Edinburgh, Edinburgh, UK

${ }^{*}$ C.J.O’Grady@sms.ed.ac.uk

Chapter in Oxford Handbook of Psycholinguistics (Second Edition), edited by Shirley-Ann Rueschemeyer and M. Gareth Gaskell

\section{Abstract and Keywords}

Models of Language Evolution reviews the models that provide evidence for the role of cultural evolution in the emergence of linguistic structure. This chapter discusses the levels of linguistic structure, and why the emergence of structure in language is a central question for evolutionary linguistics. It reviews the computational and experimental models which demonstrate that pressures operating during language learning and language use can give rise to the appearance of design in language, through the repeated cycle of learning and use that characterise language transmission. Finally, it discusses how learning biases at the individual level lead to the presence of typological universals: systematic patterns in how the world's languages tend to be structured.

Keywords: language evolution, combinatoriality, compositionality, cultural transmission, iterated learning 


\section{Introduction}

Language is startling in its complexity and expressive power. Unlike any other animal communication system, language provides a system for building complex signals from sub-components in a way that yields an endless set of possible combinations, capable of conveying an infinite array of possible meanings.

This open-ended expressivity arises through duality of patterning (Hockett, 1960), which is the capacity of language to combine simpler structures to create more complex structures, at two distinct levels. At the first level, language combines meaningless units (that is, phonemes) into meaningful words and morphemes. For example, consider the English phonemes /d/, /b/, and /a/. None of these units have meaning on their own, but they can be combined into different meaningful combinations, such as "bad" and "dad". This is combinatoriality, and it gives us a highly efficient and expressive system . For example, most varieties of English have 40-45 phonemes (e.g. Ladefoged (2006) lists 44 phonemes in the inventory of Received Pronunciation), which can be recombined to form the 291,500 entries in the second edition of the Oxford English Dictionary ("Dictionary Facts - Oxford English Dictionary," 2016).

At the second level of combination, meaningful words and morphemes are combined into larger, more complex meaningful units at the level of the phrase and sentence. For instance, the morpheme "bed" can be combined with the plural morpheme to create the word "beds", which in turn can be combined with other morphemes in a sentence like "The hotel room had two beds." This compositionality makes it easy for people to generalise rules they have already learned to new items they encounter. For instance, young children might learn the word "bed" and its plural "beds", the word "dog" and its plural "dogs", and so on. Then, when presented with a nonsense word "wug", they generalise what they have learned about the plural morpheme to create the word "wugs" (Berko, 1958). The same processes of generalisation apply at the level of multi-word utterances-compositional structure allows us to routinely produce and understand sentences that we have never seen (or that have never been uttered) before.

A major challenge for evolutionary linguists is to establish how a system like this can evolve, and why only humans have a communication system that works in this way. One potential explanation is that language is the result of cultural evolution (Christiansen \& Chater, 2008; Kirby, 2001). Language, in common with many other human behaviours, is culturally transmitted: we are exposed to language by listening to those around us, and based on this linguistic input, we learn that language. Then, in turn, we produce linguistic output which forms the basis for language learning in others, who pass the language on themselves, and so on. This chapter will review a growing body of evidence suggesting that this type of multi-generational transmission process (sometimes known as iterated learning: Kirby \& Hurford, 2002) forces languages to adapt to constraints that affect how humans learn and transmit languages. Both computer models and experimental work on human learning suggest that this process of cultural evolution can result in the emergence of linguistic structure. 


\section{Computational models of language evolution}

Early work investigating language evolution used computational modelling to simulate evolutionary processes acting on communication systems (Hurford, 1989; Steels, 1999). In a seminal paper, Kirby (2000) used computer simulations of iterated learning to demonstrate that compositionality could emerge in a model where each generation of computational agents learned a communication system from linguistic data produced by a previous generation that had learned in the same way. Kirby initialised his simulations with holistic systems (where meanings are communicated by signals that have no internal structure); as this 'language' was passed from generation to generation, with each generation of learners searching for generalisations in the data they were presented with, compositional structure gradually developed, until after many hundreds of generations an elegant, compositional language had formed, where signals are composed through the rule-governed recombination of meaningful subcomponents.

An important finding of this modelling work is the role played by learning bottlenecks in iterated learning systems (Brighton, Smith, \& Kirby, 2005; Kirby, 2000, 2002; Zuidema, 2003). These bottlenecks appear between generations, as one generation of language users presents the next generation of users with only a limited set of linguistic data, on the basis of which they are required to learn an open-ended expressive system. As languages are repeatedly transmitted through a series of bottlenecks, compositional structure develops.

The role of the bottleneck in driving the emergence of structure can be best illustrated through an example. Imagine a virtual world with moving shapes of various colours: there are squares, circles and triangles, which can be red, blue or green, and move in spirals, bounces, or straight lines. If each possible referent in this world is expressed with a holistic signal (that is, one signal for a blue square that bounces, another completely distinct signal for a blue square that spirals, yet another unrelated signal for a red square that spirals, etc.), twentyseven signals would be required in order to express the full range of referents. If a learner is only exposed to some of the twenty-seven words (perhaps half of them) , they have no way to accurately reconstruct the 'correct' signals for referents they haven't seen labelled, because there is no pattern underlying how labels are associated with referents.

However, if the communication system is compositional, twenty-seven meanings can be expressed using only nine signals (three signals expressing colour, three shape, and three expressing motion) and one rule for combining them (for example, combine them in the order colour-motion-shape). In this case, a learner does not need exposure to all twenty-seven labels in order to learn the full language, but instead can infer the underlying system from a smaller number of observations (for an optimal generaliser, simply encountering each of the 9 component parts once is sufficient), and subsequently exploit the compositional structure to produce labels which they didn't encounter while learning. 
Whenever there is a learning bottleneck, language learners are forced to generalise, because the input they receive does not provide labels for all the meanings they might like to convey. This forces learners to hunt for commonalities across holistic labels (for instance observing that the syllable "ka" appears on two bouncing shapes, and then using it for all future bouncing shapes). Over time, these generalisations accumulate, and holistic languages gradually change to become compositional.

This seminal modelling work has been built on in two important directions. A related body of work focuses on phonological systems, showing that interaction and cultural transmission can explain how the phonemes found in the world's languages are organised in acoustic and articulatory space, and how combinatorial sound systems emerge (De Boer, 2000, 2001; Oudeyer, 2005, 2006; Wedel, 2006, 2012; Zuidema \& de Boer, 2009). Another recent body of computational and mathematical modelling work has built on the earlier simulation-based results to extract general principles for how iterated learning and transmission bottlenecks shape linguistic systems (Griffiths \& Kalish, 2007; Kirby, Dowman, \& Griffiths, 2007; Perfors \& Navarro, 2014), and to explore how cultural and biological evolution interact to shape languages and language learners (Smith \& Kirby, 2008; Thompson, Kirby, \& Smith, 2016).

\section{Language evolution in the lab}

Computational and mathematical models continue to play a vital role in the emerging study of cultural evolution as it applies to language. However, scepticism regarding the applicability of these models to human language learners necessitates supplementing computational work with experiments using human participants. Experiments on cultural evolution are based on the same principal as models described above: they explore how data transmitted across generations of individuals is shaped by the process of transmission. In an experimental setting, this method of creating "transmission chains" of participants has been highly successful in understanding cultural evolutionary processes more generally (Whiten, Caldwell, \& Mesoudi, 2016). In a standard transmission chain experiment, the first participant in a chain is presented with some material (e.g. a drawing to copy, or a miniature language to learn) and then required to reproduce it. This reproduction is then used as the training material for the next participant in the chain of transmission, and so on.

Importantly, each participant in a chain of transmission is not simply reproducing the material they are given, but rather forming a mental representation of it, and then using this mental representation to reproduce the material they were earlier presented with (Mesoudi \& Whiten, 2008). Because of this process of internalisation and recall, if participants have any pre-existing cognitive biases or expectations about the material, those biases will be imposed on their representation and subsequent recreation of the material, allowing for a transformation of the material as it is passed from person to person (Griffiths, Christian, \& Kalish, 2008; Kalish, Griffiths, \& Lewandowsky, 2007). 
The earliest study done using transmission chains, called "serial reproduction" (Bartlett, 1932), found that the contents of certain genres of stories were transmitted more fully than others, and that story contents became distorted over time to match participants' pre-existing knowledge. More recent studies have used the method to study cultural change, finding that social information such as gossip is transmitted more accurately than non-social information (Mesoudi, Whiten, \& Dunbar, 2006).

Cultural transmission therefore entails two vital steps: first learning the information, and then reproducing it. An important question, then, is which of these two processes is responsible for the changes that occur to systems that are transmitted through iterated learning. Tamariz \& Kirby (2015) manipulated these pressures in a simple drawing task: participants in transmission chains were asked to look at a drawing created by the previous participant in the chain and reproduce it. In one set of chains, each participant was given time to memorize the drawing, and then reproduced it from memory; in the other set, participants were able to reproduce the drawing directly, while looking at it and without having to memorize it first. In the chains where participants had to memorize the drawing before reproducing it, the drawings became smaller and less complex over successive generations, and began to tend towards conventional cultural symbols such as numbers. In the chains that simply copied the drawing directly, there was no decrease in size or complexity, and no tendency towards symbolism. This suggests that learning, rather than reproducing, is what creates the bias towards compressibility.

These transmission chain methods therefore allow researchers to establish what kinds of information are best retained and transmitted, and infer what systemic biases might be at play during transmission (Mesoudi \& Whiten, 2008). Taken together, the growing list of biases can help to explain current cultural phenomena, such as religion, music-and language (Chater \& Christiansen, 2010).

\subsection{Learnability and linguistic structure}

Applying these techniques to artificial languages or communicative games allows us to investigate how communication systems evolve through cultural transmission, providing a close experimental analogue to the computational models reviewed in Section 2. In an early paper directly inspired by this modelling work, Kirby et al. (2008) taught participants a series of randomlygenerated holistic labels for the set of 27 shapes described in Section x.2 (27 shapes generated by combining three colours, three shapes, and three kinds of motion). After the training phase, participants were required to provide labels for these shapes, with their output used as the training input for the next participant in the chain. The transmission chain entailed a learning bottleneck between each generation: each participant was trained only on labels for a subset of the total set of shapes (14 of the 27), but was required to produce labels for the full set. This forced participants to provide labels for shapes they had never seen the label for, introducing a strong pressure for generalisation 
(although many did not realise that many of the test stimuli were previously unseen).

Although the labels provided to the first generation in each chain were random, the artificial languages evolved over the transmission chains to become regular and generalizable. In an initial experiment, the labels became highly simplified (e.g. "poi" for anything moving in a spiral pattern, regardless of shapes or colour). Languages like this are highly learnable - there simply isn't much to learn - but not very useful for communicating, because each label would drastically underspecify its intended referent (e.g. "poi" would fail to distinguish between a spiralling black triangle and a spiralling red circle). In a second experiment, Kirby et al. (2008) manipulated each participant's output before passing it on to the subsequent learner in the chain, removing duplicate labels from each participant's test answers. This experimental manipulation was intended to mimic the natural pressure, acting on real languages, to be useful for communication. ${ }^{1}$ In this second experiment, the languages evolved over repeated episodes of transmission and developed compositional structure: subcomponents of each label specified sub-components of the stimulus. For example, the first syllable might specify the colour, the second the shape, and the third syllable the kind of movement. This compositional structure makes the language simple enough for experimental participants to learn fairly accurately, while by-passing the ambiguity filter by providing every object with a unique label.

Subsequent work shows that a similar result is obtained if this artificial prohibition is replaced by actual communicative interaction. Kirby et al. (2015) ran an iterated learning experiment where pairs of participants were trained on an artificial language and then used it to communicate, taking turns to label pictures for each other - ambiguous labels would be problematic during this communicative task, but were not prohibited. The language produced during communication was then passed on to a fresh pair of participants, who in turn learned the language and used it to communicate, and so on. As expected, this repeated process of learning and use resulted in the gradual emergence of compositional structure. Kirby et al. (2015) also showed that the development of structure is dependent on transmission: when a single pair of participants play the same communication game over and over, compositional structure does not develop, showing that both learning and use play crucial roles.

Similar techniques have been used to explore the emergence of combinatoriality. As discussed in the introduction, combinatoriality allows us to use a few 10 s of speech sounds to generate tens of thousands of meaningful words. Hockett (1960) suggested that combinatoriality might therefore be a consequence of a pressure to create a large number of distinct meaningful signals - that is, as the potential number of meanings in a communication system grows, and it becomes more difficult to create new holistic signals, the communication system might

\footnotetext{
1 Similar pressures were included in the computational models outlined in section 2 , e.g. in the form of a prohibition on pairing multiple referents with a single ambiguous label, again as a proxy for pressures presumably arising from communication in the real-world case.
} 
begin to re-use components of the holistic signals in a combinatorial fashion. However, at least one human language doesn't use combinatoriality to solve this problem. Al-Sayyid Bedouin Sign Language (ABSL), an emerging sign language, is still in the process of developing structure at the phonological level (which in the case of sign languages is achieved by handshapes that are meaningless on their own but combined into meaningful units) (Israel \& Sandler, 2011; Sandler, Aronoff, Meir, \& Padden, 2011). Despite lacking phonemes, ABSL is nonetheless capable of expressing a wide array of meanings, and is used for all the functions that other human languages are used for. This suggests that a fully-functional language can develop a large meaning space without combinatoriality, implying that a growing meaning space alone is not sufficient pressure for combinatoriality to emerge.

Verhoef et al. (2011) used an iterated language learning experiment to show instead that the pressure of learning holistic signals can, through cultural transmission, explain the emergence of combinatoriality. In an experiment in which participants are required to learn and then reproduce a sequence of twelve slide whistle sounds - which are holistic in the first generation of the chain, i.e. with no internal structure, simply random and continuous slidewhistle movements/sounds-they show that the whistle sounds begin to show structure, with internal components of the holistic signals beginning to be reused within and between different sounds in the sequence. As a result, the sequence becomes more easily learnable and more accurately transmitted. This suggests that combinatorial structure can emerge independently of pressures arising from the number of meanings which need to be conveyed.

This finding is corroborated by Roberts \& Galantucci (2012), who used a communication game to compare the effects of conventionalization and number of meanings on the emergence of combinatoriality. Participant pairs, separated from each other at separate computers, were presented with a grid with simple animal drawings, and had to communicate to each other which drawing to select. Communication was possible using a stylus that distorted their drawings to prevent participants from simply sketching the animal to be communicated. Conventionalization over the course of this repeated drawing game resulted in the emergence of drawings which exhibited combinatorial structure (subelements of drawings that were repeated across drawings), but the number of meanings was only weakly correlated with combinatoriality, which the authors suggest may be due to the limitations on the number of meanings that could be used in the experiment (in this case, only 20).

Properties of the communication medium may also contribute to the emergence of combinatoriality, namely rapid fade. Signals in spoken and signed language (and in Verhoef et al.'s slide whistle experiment) are transient: signals linger for only a short period of time (Hockett, 1960). Galantucci, Kroos, \& Rhodes (2010), using a graphical communication task similar to that employed by Roberts \& Galantucci (2012), manipulated the rapidity of fade of signals by changing the speed with which communicative drawings faded from the screen. They found that more rapid fading led to a higher degree of combinatoriality. The number of meanings to be communicated was again not related to the degree of 
combinatoriality, providing further evidence against the hypothesis that larger meaning spaces drive the emergence of combinatoriality.

\subsection{Communication and the emergence of iconicity}

Transmission chain experiments such as these are therefore capable of explaining how linguistic structure arises. Perhaps surprisingly, similar methods have also provided insights into the very nature of linguistic signals, namely the fact that they are composed of arbitrary symbols for conveying concepts.

Garrod, Fay, Lee, Oberlander, and Macleod (2007) use a Pictionary-like task involving a list of easily confused concepts-such as "art gallery", "museum", "parliament" and "theatre" — presented to a pair of participants. The Drawer in each turn must draw a randomly selected word from the list, while the Matcher must attempt to guess which of the words on the list the director is attempting to draw. As each pair of participants repeatedly plays this game, drawing the same concept multiple times, their drawings gradually become simpler and more abstract, and partners playing together converge, producing more similar drawings for a given concept. For instance, "cartoon" in one pair was initially drawn as a cartoon bunny and a bird, and over six rounds, simplified to become just a pair of stylized bunny ears. These results illustrate how iconic representations-representations that depict the content being communicated through resemblance-become abstract and symbolic through repeated use (Garrod et al., 2007), linked to their meaning only by convention.

Interaction appears to be an essential component of this process: transmission alone does not result in either convergence on shared representations or simplification (Garrod, Fay, Rogers, Walker, \& Swoboda, 2010). However, in an experiment that combined the community-based game with a transmission chain, by periodically removing the most experienced member of the group and replacing them with a naïve participant, drawings became symbolic to the extent that newcomers were required to learn their community's conventionalized symbols for the list of concepts (Caldwell \& Smith, 2012). A similar effect is found when a drawing task occurs in a community-like setting that has eight participants interacting in a closed circle of shuffled pairs (Fay, Garrod, Roberts, \& Swoboda, 2010).

These graphical communication paradigms show how symbolic, arbitrary systems arise through repeated interaction (whether in a pair or community), while the iterated learning paradigm shows how arbitrary systems become systematic through repeated learning. Theisen, Oberlander and Kirby (2009) and Theisen-White, Kirby and Oberlander (2011) combine the two paradigms to demonstrate how the arbitrary symbols come to be used systematically, as they are in language. These studies use a graphical communication task with the potential for compositionality, by creating a list of items with shared semantic features: for example, five different kinds of entity (like people or buildings) through ten different themes (like education). Thus, the person in the education theme would be a teacher (Theisen et al., 2009). 
As in previous experiments in the graphical communication paradigm, Theisen et al. (2009) found that drawings become increasingly arbitrary over time, while the arbitrary elements came to be used systematically: over repeated interactions, pairs of participants began to use increasingly symbolic elements to indicate components of the meanings they were communicating. For instance, if the first instance of "teacher" in a pair resulted in a drawing of a blackboard, subsequent school-related drawings ("teacher", but also "school" and "school bus") for that pair might include a simplified chalkboard element, plus a second simple component specifying which school-related concept was being conveyed. When a transmission chain was added, by using the first generation's drawings as training material for a second generation of interlocutors, the level of systematicity increased (Theisen-White et al., 2011). This suggests that both horizontal interaction and vertical transmission play a role in creating arbitrary and compositional systems, and that both communicative utility and learnability (by new players in the game) play an essential role in the emergence of structure, mirroring the results discussed above from Kirby et al. (2015).

\subsection{Regularity and systematicity}

The studies reviewed above focus on how languages and other communication systems are shaped by pressures inherent in their transmission - the requirements of learners to produce utterances for new meanings, or to learn and reproduce sets of rapidly-fading signals. A related strand of work (underpinned by a series of mathematical and computational models, e.g. Griffiths \& Kalish, 2007) explores how biases of learners, rather than external features of the transmission process, might also contribute to shaping language evolution. In particular, an intriguing body of work shows that even very weak biases in learning can have large effects on how languages are structured, because those weak biases accumulate over generations to create a substantial effect.

Some of the experimental work showing that this is the case has been concerned with the evolution of linguistic variation. Languages provide language users with multiple roughly equivalent possibilities for particular forms, e.g. allophones (such as dark or light /l/), allomorphs (e.g. the past tense "-ed" is pronounced differently on the words "jumped" and "dragged"), or synonyms. The variant that is deployed in any given situation tends to be fairly predictable, being conditioned on sociolinguistic, phonological, semantic, or other criteria (Givon, 1985).

Naively, we might therefore expect that the conditioned, predictable nature of variation in language therefore reflects a strong bias in language learning, strongly predisposing learners to condition or eliminate unpredictable variation wherever it occurs. Counterintuitively, adults appear to show no such tendency: if trained on unpredictable language data, the instead tend to probability match. For instance, if a particular variant appears $70 \%$ of the time in their training data, they tend towards using it at roughly the same rate and in a similarly 
unconditioned fashion (Hudson Kam \& Newport, 2005; Wonnacott \& Newport, 2005). This means that, in individual language learners, variant forms are preserved and remain unpredictable.

However, Reali and Griffiths (2009) show that this picture changes when unpredictably variable linguistic systems are passed along transmission chains. Using an approximation of synonymy in natural language, participants in their experiment were presented objects paired with labels. Each participant saw two labels for each object, with the two labels appearing with varying probability (e.g. for one object the two labels might appear in a 50-50 ratio, for another object the ratio of the labels might be 80-20). After training, when participants were asked to repeatedly label each object, single learners showed only a weak tendency towards regularization, essentially matching the probability distribution of the input. However, across transmission chains, where these systems of object labelling were passed from person to person, there was a strong tendency towards regularization, resulting in the loss of one of the variants.

One explanation for this result is that transmission chains automatically bring about the elimination of variation. However, Smith and Wonnacott (2010) find that variation can be maintained in a transmission chain, if that variation can become conditioned. In their experiment, adult learners learned and attempted to reproduce a variable system of plural markers - they learned a language in which the plural could be marked in one of two ways, with both forms occurring completely unpredictably. In line with the results from Reali \& Griffiths (2009) individual learners did not exhibit a detectable tendency towards eliminating or conditioning this variation. However, after these miniature languages had been transmitted down chains, the variation was preserved (both plural markers lived on), but became predictable - each plural marker gradually became associated with a subset of the nouns, such that some nouns always took one plural marker and other nouns took the other. In their experiment conditioning on the noun was the only possible way for the variation to become conditioned; in real languages, multiple such contexts exist, allowing for potentially complex but ultimately predictable systems of conditioned variation.

Collectively, these results provide reason for caution in extrapolating from individual-level experiments to assumptions about the emergence of linguistic features-finding no, or limited, evidence of a bias in a single generation of learners does not imply that the bias would not emerge as a factor on a population level.

\subsection{Typological universals}

Some features of linguistic structure are common to all (in the case of compositionality and systematicity), or virtually all (in the case of combinatoriality) of the world's languages. There are also many other features in which languages vary, for instance in the order in which words are typically combined. However, even here there are significant tendencies towards 
particular features, with multiple languages appearing to converge on the same structural solutions.

For instance, basic word order rules governing the sequence of subjects $(S)$, objects $(\mathrm{O})$ and verbs $(\mathrm{V})$ in sentences could logically result in six different combinations (SOV, SVO, VSO, VOS, OSV, OVS). However, most of the world's languages use either SVO or SOV order. Although historical relationships between languages are likely to explain a number of these statistical tendencies (e.g. (Dunn, Greenhill, Levinson, \& Gray, 2011)), a new and growing body of experimental work suggests that biases in learning are also likely to play a role in explaining such tendencies, in word order (Culbertson, Smolensky, \& Legendre, 2012), morphological encoding of information (Fedzechkina, Jaeger, \& Newport, 2012) and phonological patterning (Wilson, 2003).

Experiments using silent gesture paradigms are ideal for investigating questions like these, by allowing us to see which ordering strategies participants use when communicating in a novel medium. In these experiments, participants with no experience in any sign language are required to use silent gesture to express propositions, often as part of a communicative game, a little like the parlour game Charades. In these experiments, silent gesturers show a preference for SOV word order - for instance, when presented with a picture of a pirate throwing a guitar, a participant would gesture "pirate", "guitar" and "throw", in that order. This preference appears regardless of the dominant word order in participants' native languages (Goldin-Meadow, So, Ozyurek, \& Mylander, 2008).

However, although this might explain the prevalence of SOV in the world's languages, it cannot explain the prevalence of SVO. Schouwstra and de Swart (2014) show that the semantic content of a message results in different word orders in silent gesture. Specifically, extensional verbs that describe the relationship between specific and existent entities, such as "throw" or "kick", are expressed with a preference for SOV word order. Intensional verbs like "imagine", which take objects that may be non-existent or non-specific, are expressed with a preference for SVO word order. Both preferences hold true regardless of whether a participant's native language uses SVO or SOV.

While these results go some way towards explaining why both SOV and SVO are common word orders in the world's languages, they are unable to explain why individual languages are usually consistent in their use of either SOV or SVO, rather than conditioning word order on the semantics of the verb. This tendency for consistency can be explained by cultural transmission. Schouwstra et al., (2016) combined the silent gesture task with a transmission chain approach, where groups of participants played a Charades-like communicative game while new group members gradually replaced more experienced individuals. While at the early stages each group's system of gestures showed a mix of SOV and SVO, conditioned on verb semantics, over time, word order became conventionalised to either all-SOV or all-SVO, as in earlier transmission chain experiments with artificial languages that found a tendency towards regularization (Reali \& Griffiths, 2009; Smith \& Wonnacott, 2010). 
More fine-grained typological patterns also appear to be mirrored in the biases of language learners. For instance, the majority of languages exhibit harmonic patterns governing the ordering of adjectives and numerals that modify nouns (Greenberg, 1963): most of the world's languages have both post-nominal adjectives and numerals, or pre-nominal adjective and numerals (as in English: "blue cup", "three cups"), rather than a mix of pre- and post-nominal modifiers.

Culbertson et al. (2012) presented adult participants with an artificial language that had either mainly harmonic (adjectives and numerals both appeared pre- or post-nominally) or non-harmonic (adjectives appeared pre-nominally, numerals post-nominally, or the reverse) ordering, with a scattering of other word orders. Participants trained on languages which were mainly harmonic tended to make the language more harmonic, reducing the proportion of 'noisy' other orders. However, participants trained on mainly non-harmonic patterns behaved differently, either simply maintaining a mix of orderings, or failing to reproduce the dominant non-harmonic patterns entirely. This suggests that the crosslinguistic preference for harmonic orders, as well as the scarcity of certain nonharmonic orders in the world's languages, may arise from learning biases on the part of language learners. Subsequent work has shown the presence of similar but stronger biases in child learners (Culbertson and Newport (2015)), and other typological patterns (Culbertson \& Adger, 2014).

\section{Cross-cultural comparisons and biological evolution}

Computational and experimental models of language evolution have shown how iterated learning is capable of generating compositionality, combinatoriality, and regularity in language; how communication interacts with cultural transmission to create an expressivity pressure that contributes to the emergence of structure; and how artificial language learning paradigms can illuminate the biases that are related to typological universals.

However, there are still a number of open questions in the field. Most notably, cultural transmission is not a process unique to humans: many species have culturally-transmitted repertoires of behaviour, and there are even other species who exhibit communicative behaviours that are culturally transmitted. Bird song, for example, is culturally transmitted in many species - birds learn their song early in development through exposure to species-typical song input. These systems have striking parallels with language: they have combinatorial (albeit not compositional or semantic) structure (Berwick, Okanoya, Beckers, \& Bolhuis, 2011); and although isolated songbirds do not acquire a fully-fledged system, but rather a degenerate and simplified one, this degenerate isolate song can revert to wild-type 'natural' song when passed through a transmission chain of songbirds (Fehér, Wang, Saar, Mitra, \& Tchernichovski, 2009). This suggests the existence of learning biases that shape the evolution of song (in zebra finches, and presumably in other species), much in the same way that human learning biases and cultural evolution shape the evolution of language (Claidière, Smith, Kirby, \& Fagot, 2014; Kirby, Griffiths, \& Smith, 2014). 
These findings cohere with the models presented in this chapter, which predict the emergence of structure in an expressive system that is culturally transmitted (Kirby et al., 2015; Verhoef et al., 2011). Crucially, the nature of the expressivity pressure in birds is substantially different from that found in humans: whereas humans use signals to differentiate between possible referents in a communicative context, songbirds express mate quality through the size of their signal repertoire (Collins, 2004). Similarly, many species have a small repertoires of unlearned, holistic referential signals (for example, vervet monkeys have a small set of alarm calls signalling different kinds of predators) (Seyfarth, Cheney, \& Marler, 1980), which is consistent with the prediction that communication systems emerging without a pressure for learnability will be holistic, with no compositional or combinatorial structure. Comparative studies investigating the similarities and differences between human communication and cultural transmission, and similar systems found in other species, are likely to continue be a fruitful area of investigation for better understanding of our own cognition.

Finally, some accounts of the evolution of human language have argued that language is underpinned by language-specific cognitive apparatus, which has evolved through evolution by natural selection because the ability to communicate in this open-ended way is adaptive (e.g. Pinker \& Bloom, 1990). This suggests that linguistic structure is the result of biological adaptation, a reflection of a human-unique capacity for and predisposition to acquire communication systems with these properties. The evidence reviewed in this chapter suggests that cultural evolution is capable of explaining linguistic structure without appealing to additional, biological mechanisms: the processes involved in language learning and use are sufficient to result in the evolution of structure. But this is not to say that biological evolution should be ignored: a comprehensive account of language evolution will also need to explain the biological adaptations that led to our particular cultural environment, cognitive biases, and ability to learn complex signalling systems.

\section{References}

Bartlett, F. C. (1932). Remembering. Cambridge University Press.

Berko, J. (1958). The child's learning of English morphology. Word, 14(2-3), 150177.

Berwick, R. C., Okanoya, K., Beckers, G. J. L., \& Bolhuis, J. J. (2011). Songs to syntax: The linguistics of birdsong. Trends in Cognitive Sciences, 15(3), 113121. http://doi.org/10.1016/j.tics.2011.01.002

Brighton, H., Smith, K., \& Kirby, S. (2005). Language as an evolutionary system. Physics of Life Reviews (Vol. 2). http://doi.org/10.1016/j.plrev.2005.06.001

Caldwell, C. A., \& Smith, K. (2012). Cultural evolution and perpetuation of arbitrary communicative conventions in experimental microsocieties. PLoS ONE, 7(8). http://doi.org/10.1371/journal.pone.0043807 
Chater, N., \& Christiansen, M. H. (2010). Language Acquisition Meets Language Evolution. Cognitive Science, 34(7), 1131-1157. http://doi.org/10.1111/j.1551-6709.2009.01049.x

Christiansen, M. H., \& Chater, N. (2008). Language as shaped by the brain. The Behavioral and Brain Sciences, 31(5), 489-508; discussion 509-58. http://doi.org/10.1017/S0140525X08004998

Claidière, N., Smith, K., Kirby, S., \& Fagot, J. (2014). Cultural evolution of systematically structured behaviour in a non-human primate. Proceedings. Biological Sciences / The Royal Society, 281(1797), 20141541. http://doi.org/10.1098/rspb.2014.1541

Collins, S. (2004). Vocal fighting and flirting: The functions of birdsong. In P. Marler \& H. Slabbekoorn (Eds.), Nature's music: The science of birdsong. Academic Press.

Culbertson, J., \& Adger, D. (2014). Language learners privilege structured meaning over surface frequency. Proceedings of the National Academy of Sciences of the United States of America, 1-6. http://doi.org/10.1073/pnas.1320525111

Culbertson, J., \& Newport, E. L. (2015). Harmonic biases in child learners: In support of language universals. Cognition, 139, 71-82. http://doi.org/10.1016/j.cognition.2015.02.007

Culbertson, J., Smolensky, P., \& Legendre, G. (2012). Learning biases predict a word order universal. Cognition, 122(3), 306-29. http://doi.org/10.1016/j.cognition.2011.10.017

De Boer, B. (2000). Self-organization in vowel systems. Journal of Phonetics, 28(4), 441-465.

De Boer, B. (2001). The Origins of Vowel Systems. Oxford University Press.

Dictionary Facts - Oxford English Dictionary. (2016).

Dunn, M., Greenhill, S. J., Levinson, S. C., \& Gray, R. D. (2011). Evolved structure of language shows lineage-specific trends in word-order universals. Nature, 473(7345), 79-82. http://doi.org/10.1038/nature09923

Fay, N., Garrod, S., Roberts, L., \& Swoboda, N. (2010). The interactive evolution of human communication systems. Cognitive Science, 34(3), 351-386. http://doi.org/10.1111/j.1551-6709.2009.01090.x

Fedzechkina, M., Jaeger, T. F., \& Newport, E. L. (2012). Language learners restructure their input to facilitate efficient communication. Proceedings of the National Academy of Sciences of the United States of America, 109(44), 17897-902. http://doi.org/10.1073/pnas.1215776109

Fehér, O., Wang, H., Saar, S., Mitra, P. P., \& Tchernichovski, O. (2009). De novo establishment of wild-type song culture in the zebra finch. Nature, 459(7246), 564-568. http://doi.org/10.1038/nature07994

Galantucci, B., Kroos, C., \& Rhodes, T. (2010). The effects of rapidity of fading on communication systems. Interaction Studies, 11, 100-111. http://doi.org/10.1017/CB09781107415324.004

Garrod, S., Fay, N., Lee, J., Oberlander, J., \& Macleod, T. (2007). Foundations of representation: where might graphical symbol systems come from? Cognitive Science, 31(6), 961-987. http://doi.org/10.1080/03640210701703659

Garrod, S., Fay, N., Rogers, S., Walker, B., \& Swoboda, N. (2010). Can iterated learning explain the emergence of graphical symbols? Interaction Studies, 
11(1), 33-50. http://doi.org/10.1075/is.11.1.04gar

Givon, T. (1985). Function, structure, and language acquisition. In D. Slobin (Ed.), The crosslinguistic study of language acquisition (volume 2) (pp. 1005-1028). Hillsdale, NJ: Lawrence Erlbaum.

Goldin-Meadow, S., So, W. C., Ozyurek, A., \& Mylander, C. (2008). The natural order of events: How speakers of different languages represent events nonverbally. Proceedings of the National Academy of Sciences of the United States of America, 105(27), 9163-9168.

Greenberg, J. H. (1963). Some universals of grammar with particular reference to the order of meaningful elements. In J. H. Greenberg (Ed.), Universals of Language (pp. 73-113). MIT Press, Cambridge, MA.

Griffiths, T. L., Christian, B. R., \& Kalish, M. L. (2008). Using category structures to test iterated learning as a method for identifying inductive biases. Cognitive Science, 32(1), 68-107. http://doi.org/10.1080/03640210701801974

Griffiths, T. L., \& Kalish, M. L. (2007). Language evolution by iterated learning with bayesian agents. Cognitive Science, 31(3), 441-480. http://doi.org/10.1080/15326900701326576

Hockett, C. F. (1960). The Origin of Speech,. Scientific American, 203, 88-111.

Hudson Kam, C. L., \& Newport, E. (2005). Regularizing Unpredictable Variation: The Roles of Adult and Child Learners in Language Formation and Change. Language Learning and Development, 1(2), 151-195. http://doi.org/10.1207/s15473341lld0102_3

Hurford, J. R. (1989). Biological evolution of the Saussurean sign as a component of the language acquisition device. Lingua, 77(2), 187-222. http://doi.org/10.1016/0024-3841(89)90015-6

Israel, A., \& Sandler, W. (2011). Phonological category resolution: A study of handshapes in younger and older sign languages. In R. Channon \& H. van der Hulst (Eds.), Formational Units in Sign Language. Mouton de Gruyter.

Kalish, M. L., Griffiths, T. L., \& Lewandowsky, S. (2007). Iterated learning: Intergenerational knowledge transmission reveals inductive biases. Psychonomic Bulletin \& Review, 14(2), 288-294. http://doi.org/10.3758/BF03194066

Kirby, S. (2000). Syntax without natural selection: How compositionality emerges from vocabulary in a population of learners. The Evolutionary Emergence of Language: Social Function and the Origins of Linguistics Form, $303-323$.

Kirby, S. (2001). Spontaneous evolution of linguistic structure - An iterated learning model of the emergence of regularity and irregularity. IEEE Transactions on Evolutionary Computation, 5(2), 102-110. http://doi.org/10.1109/4235.918430

Kirby, S. (2002). Learning, bottlenecks and the evolution of recursive syntax. (T. Briscoe, Ed.)Linguistic Evolution through Language Acquisition: Formal and Computational Models. Cambridge University Press.

Kirby, S., Cornish, H., \& Smith, K. (2008). Cumulative cultural evolution in the laboratory: An experimental approach to the origins of structure in human language. PNAS, 105(31), 10681-10686.

Kirby, S., Dowman, M., \& Griffiths, T. L. (2007). Innateness and culture in the evolution of language. Proceedings of the National Academy of Sciences, 104(12), 5241-5245. 
Kirby, S., Griffiths, T., \& Smith, K. (2014). Iterated learning and the evolution of language. Current Opinion in Neurobiology, 28, 108-114. http://doi.org/10.1016/j.conb.2014.07.014

Kirby, S., \& Hurford, J. R. (2002). The emergence of linguistic structure: An overview of the iterated learning model. Simulating the Evolution of Language. http://doi.org/10.1016/j.jpba.2005.07.044

Kirby, S., Tamariz, M., Cornish, H., \& Smith, K. (2015). Compression and communication in the cultural evolution of linguistic structure. Cognition, 141, 87-102. http://doi.org/10.1016/j.cognition.2015.03.016

Ladefoged, P. (2006). A Course in Phonetics (Fifth). Thomson Wadsworth.

Mesoudi, A., \& Whiten, A. (2008). The multiple roles of cultural transmission experiments in understanding human cultural evolution. Philosophical Transactions of the Royal Society of London. Series B, Biological Sciences, 363(1509), 3489-3501. http://doi.org/10.1098/rstb.2008.0129

Mesoudi, A., Whiten, A., \& Dunbar, R. I. M. (2006). A bias for social information in human cultural transmission. British Journal of Psychology, 97(3), 405-423. http://doi.org/10.1348/000712605X85871

Oudeyer, P.-Y. (2005). The Self-Organization of Speech Sounds. Retrieved from http://arxiv.org/abs/cs/0502086

Oudeyer, P.-Y. (2006). Self-organization in the Evolution of Speech. Oxford University Press.

Perfors, A., \& Navarro, D. J. (2014). Language evolution can be shaped by the structure of the world. Cognitive Science, 38(4), 775-793. http://doi.org/10.1111/cogs.12102

Pinker, S., \& Bloom, P. (1990). Natural language and natural selection. Behavioral and Brain Sciences, 13, 707-784.

Reali, F., \& Griffiths, T. L. (2009). The evolution of frequency distributions: Relating regularization to inductive biases through iterated learning. Cognition, 111(3), 317-328. http://doi.org/10.1016/j.cognition.2009.02.012

Roberts, G., \& Galantucci, B. (2012). The emergence of duality of patterning: Insights from the laboratory. Language and Cognition, 4(4), 297-318. http://doi.org/10.1515/langcog-2012-0017

Sandler, W., Aronoff, M., Meir, I., \& Padden, C. (2011). The gradual emergence of phonological form in a new language. Natural Language and Linguistic Theory, 29(2), 503-543. http://doi.org/10.1007/s11049-011-9128-2

Schouwstra, M., \& de Swart, H. (2014). The semantic origins of word order. Cognition, 131(3), 431-436. http://doi.org/10.1016/j.cognition.2014.03.004

Schouwstra, M., Smith, K., \& Kirby, S. (2016). From natural order to convention in silent gesture. In O. F. \& T. V. SG Roberts, C Cuskley, L McCrohon, L BarcelóCoblijn (Ed.), The Evolution of Language: Proceedings of the 11th International Conference (EVOLANG11). EVOLANG XI, New Orleans, United States, 20-24 March.

Seyfarth, R. M., Cheney, D. L., \& Marler, P. (1980). Monkey responses to three different alarm calls: evidence of predator classification and semantic communication. Science (New York, N.Y.), 210(4471), 801-803. http://doi.org/10.1126/science.7433999

Smith, K., \& Kirby, S. (2008). Cultural evolution: implications for understanding 
the human language faculty and its evolution. Philosophical Transactions of the Royal Society B: Biological Sciences, 363(1509), 3591-3603.

Smith, K., \& Wonnacott, E. (2010). Eliminating unpredictable variation through iterated learning. Cognition, 116(3), 444-9. http://doi.org/10.1016/j.cognition.2010.06.004

Steels, L. (1999). The Talking Heads Experiment. Laboratorium.

Tamariz, M., \& Kirby, S. (2015). Culture: Copying, compression, and conventionality. Cognitive Science, 39(1), 171-183. http://doi.org/10.1111/cogs.12144

Theisen-White, C., Kirby, S., \& Oberlander, J. (2011). Integrating the horizontal and vertical cultural transmission of novel communication systems. Expanding the Space of Cognitive Science :Proceedings of the 33rd Annual Meeting of the Cognitive Science Society, (2010), 956-961. Retrieved from http://palm.mindmodeling.org/cogsci2011/papers/0215/paper0215.pdf

Theisen, C. A., Oberlander, J., \& Kirby, S. (2009). Systematicity and arbitrariness in novel communication systems. In N. Taatgen \& H. van Rijn (Eds.), CogSci 2009 Proceedings (pp. 1971-1976). Cognitive Science Society.

Thompson, B., Kirby, S., \& Smith, K. (2016). Culture shapes the evolution of cognition. Proceedings of the National Academy of Sciences, 113(16), 45304535.

Verhoef, T., Kirby, S., \& Padden, C. (2011). Cultural emergence of combinatorial structure in an artificial whistled language. In T. Carlson, L.; Hölscher, C.; Shipley (Ed.), Proceedings of the 33rd annual conference of the cognitive science society. Austin, TX: Cognitive Science Society (pp. 483-488).

Wedel, A. (2006). Exemplar models, evolution and language change. The Linguistic Review, 23, 247-274.

Wedel, A. (2012). Lexical contrast maintenance and the organization of sublexical contrast systems. Language and Cognition, 4(4), 319-355. http://doi.org/10.1515/langcog-2012-0018

Whiten, A., Caldwell, C. A., \& Mesoudi, A. (2016). Cultural diffusion in humans and other animals. Current Opinion in Psychology, 8, 15-21. http://doi.org/10.1016/j.copsyc.2015.09.002

Wilson, C. (2003). Experimental Investigation of Phonological Naturalness. West Coast Conference on Formal Linguistics 22 (WCCFL22), 101-114.

Wonnacott, E., \& Newport, E. L. (2005). Novelty and Regularization: The Effect of Novel Instances on Rule Formation. BUCLD 29: Proceedings of the 29th Annual Boston University Conference on Language Development, 1-11.

Zuidema, W. (2003). How the poverty of the stimulus solves the poverty of the stimulus. In O. K. Becker S, Thrun S (Ed.), Advances in Neural Information Processing Systems 15 (Proceedings of NIPS'02 (Vol. 1, pp. 43-50). Cambridge, MA: MIT Press. http://doi.org/10.1017/CB09781107415324.004

Zuidema, W., \& de Boer, B. (2009). The Evolution of Combinatorial Phonology. Journal of Phonetics, 37, 125-144. 